\title{
Fiber Bragg grating sensor based on cantilever structure embedded in polymer 3D printed material
}

\author{
Rita Lima $^{* a}$, R. Tavares ${ }^{c}$, S. O. Silva ${ }^{a}$, P. Abreu ${ }^{c}$, Maria T. Restivo ${ }^{c}$, and O. Frazão ${ }^{a, b}$ \\ ${ }^{a}$ INESC-TEC, Rua do Campo Alegre 687, 4169-007 Porto, Portugal \\ ${ }^{b}$ Faculty of Sciences of Porto University, Rua do Campo Alegre 687, 4169-007 Porto, \\ Portugal \\ cLAETA-INEGI, Faculty of Engineering, University of Porto, Rua Dr. Roberto Frias, \\ 4200-465 Porto, Portugal
}

\begin{abstract}
A cantilever structure in 3D printed based on a fiber Bragg grating (FBG) sensor embedded in polymer material is proposed. The FBG sensor was embedded in 3D printed coating and was tested under three physical parameters: displacement, temperature and vibration. The sensor was tested in displacement in two different regions of the cantilever, namely, on its midpoint and end point. The maximum displacement sensitivity achieved was $(3 \pm 0.1) \mathrm{pm} / \mathrm{mm}$ for end point displacement, and a temperature sensitivity of $(30 \pm 1) \mathrm{pm} /{ }^{\circ} \mathrm{C}$ was also attained. In the case of vibration measurements it was possible to obtain a $10.23 \mathrm{~Hz}-$ low frequency oscillation.
\end{abstract}

Keywords: Fiber Bragg Grating, 3D printing, displacement sensor, temperature sensor, vibration sensor

\section{INTRODUCTION}

$3 \mathrm{D}$ printing is revealing more efficient and inexpensive applications, a lot of them in sensors' coatings, due to flexibility and resistance improvements. Between afford new optical geometries and printing multiple materials in a single optical element, the advantages are remarkable. Depending on the material extruded, different properties can be defined: size, color, shape, single or multiple materials for a single optical element and, due to the layer by layer process, print structures inside structures [1].

The fiber Bragg grating (FBG) is a microstructure written in the fiber core and it has been applied in different applications. The modification of the period or the effective index of the Bragg grating are intrinsic properties when physical parameters are applied. This sensing element can be easily included in a 3D printed part (sensor housing). The main advantage is to protect the FBG, making this sensor robust. When subjected to some physical parameter, for instance strain, pressure or displacement, the Bragg wavelength is shifted due to the deformation suffered. [2, 8]. There are a large number of applications for this promising technology in a wide number of areas, from optics to medicine. It is possible to use optical elements to fabricate interactive devices that sense user inputs (push, rotation, acceleration), such as light pipes, mobile touch sensing and diverse interactive displays. These are controlled by analyzing the fabrication process, adjusting resolutions and printer definitions, and choosing materials according to the desired final proprieties. The implementation of 3D printed sensors housing takes advantage on the flexibility and reduced cost of the production process. There is no need of molds or tools, it is an automated process and the wasted material can be recycled. The stiffness of the sensor head is also adjustable by the stiffness of its housing which can be defined during the deposition process. These advantages lead 3D-printing to be a wanted process in the fabrication of custom products. $[9,10]$.

This work presents a cantilever structure based on a FBG embedded in 3D printed polymer material. The FBG sensor was tested in terms of displacement, temperature and vibration. The displacement causes a direct stretching in the cantilever, which indirectly stretches the FBG. 


\section{3D PRINTED FABRICATION PROCESS}

The sensor is a Bragg grating embedded in a 3D printed polymer (Fig.1a), with center wavelength at $1550 \mathrm{~nm}$. The polymer used for coating has a melting point near $80^{\circ} \mathrm{C}$ and a density of $1.25 \mathrm{~g} / \mathrm{cm}^{3}$ (at $21.5^{\circ} \mathrm{C}$ ). The sensor was designed in a cantilever geometry, having a rectangular prismatic structure with $20 \times 100 \times 3 \mathrm{~mm}$ dimensions (Fig.1b), with a central structure with $5 \times 78 \times 1 \mathrm{~mm}$.

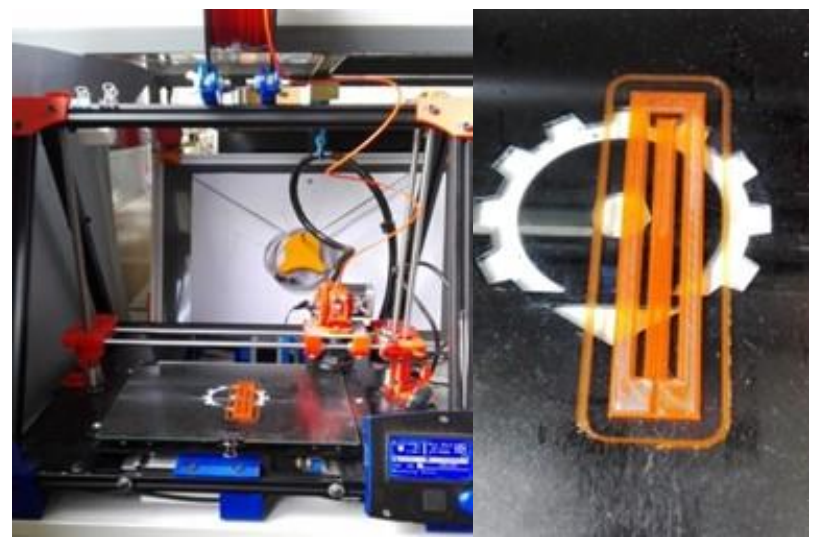

Fig. 1 a) 3D Printer fabricating the sensor. b) Fabricated sensor based on cantilever structure.

The experimental setup assembled is composed of a broadband source with bandwidth of $100 \mathrm{~nm}$ centered at $1550 \mathrm{~nm}$, an optical spectrum analyzer (OSA) and an optical circulator to interrogate the FBG in reflection. After the printing process and due to it, the center wavelength was shifted in approximately $2 \mathrm{~nm}$, to $1548.818 \mathrm{~nm}$. The registered data consists of a reflection signal from the FBG sensor, and this setup enables the OSA to acquire the signal's spectrum while testing the sensor. The data were acquired with $0.2 \mathrm{~nm}$ resolution.

\section{RESULTS}

Three physical parameters were applied to the cantilever namely displacement, temperature and vibration. The registered data were analyzed and the result is a study of the observed spectrum shift and changed properties. The displacement was applied with the help of a translation stage, with the cantilever fixed in front of it, from $0 \mathrm{~mm}$ to $5 \mathrm{~mm}$ ( $1 \mathrm{~mm}$ to $1 \mathrm{~mm}$ ) in the sensor's midpoint (Fig. 2a) and end point (Fig. 2b).

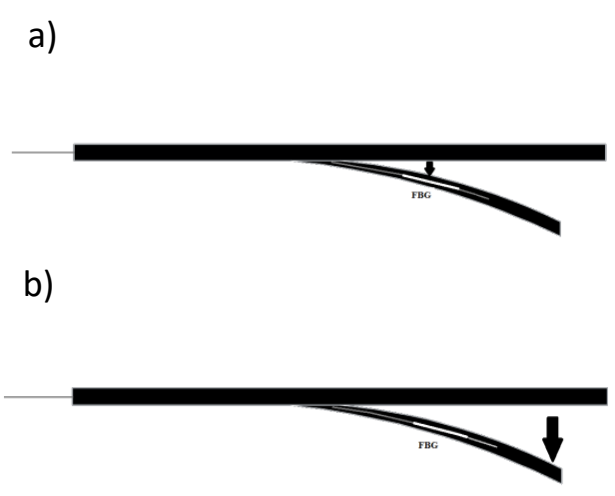

Fig. 2 Schematic of the positions where were applied the displacement, midpoint (a) and end point (b) of the sensor.

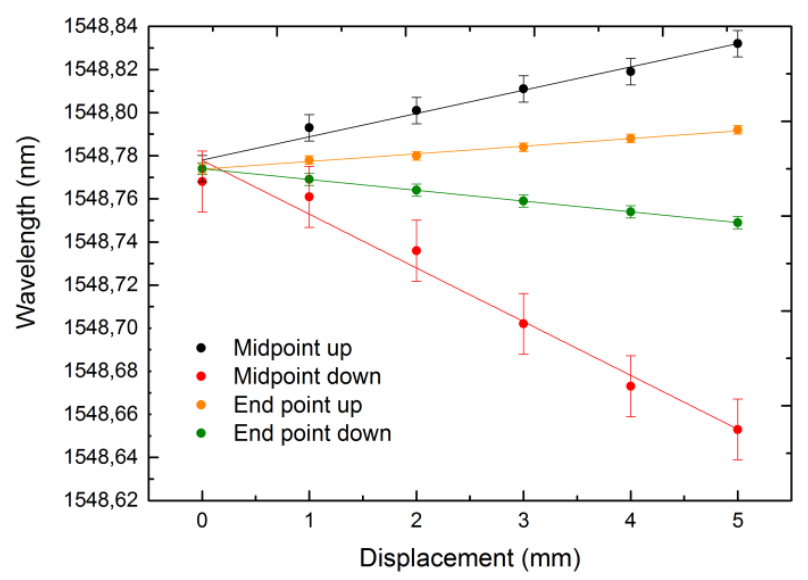

Fig. 3 Peak center wavelength response for displacement applied at the sensor's midpoint and end point. 
In Fig. 3, experimental results for displacement at the sensor's midpoint (Fig. 2a) and end point (Fig. 2b) are presented. Both displacements have linear responses and high sensitivity. For midpoint displacement the maximum sensitivity achieved was $(10 \pm 0.76) \mathrm{pm} / \mathrm{mm}$ upwards and $(20 \pm 1.0) \mathrm{pm} / \mathrm{mm}$ downward. For end point displacement the sensitivity achieved was $(3.5 \pm 0.15) \mathrm{pm} / \mathrm{mm}$ upwards and $(5 \pm 1) \mathrm{pm} / \mathrm{mm}$ downward.

The spectrum shifts to higher values if the curvature is applied upwards and to lower values if the curvature is applied downward. Both sensor and coating resisted to applied curvature successfully.

Temperature was also measured between $73.5^{\circ} \mathrm{C}$ to $38.9^{\circ} \mathrm{C}$ using an oven. The data were recorded during the cooling process. As expected, cooling causes a shift in the spectrum, which is analyzed in Fig.4, where the spectrums for two distinct temperatures are presented.

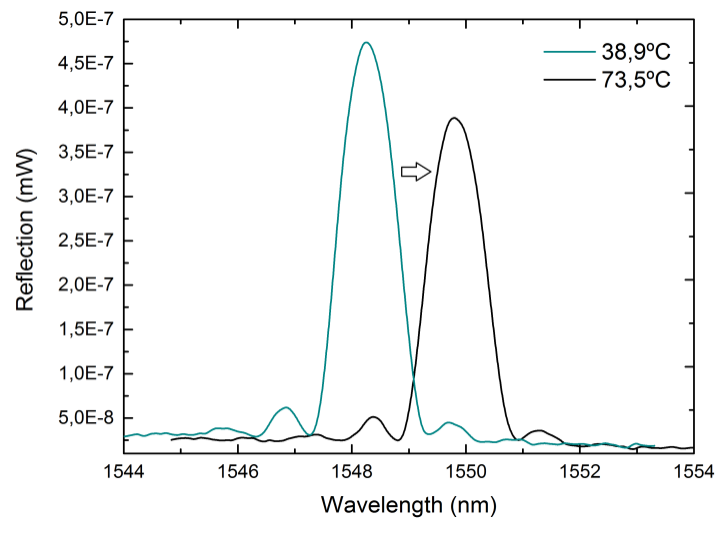

Fig 4. Shifted spectrum due to temperature.

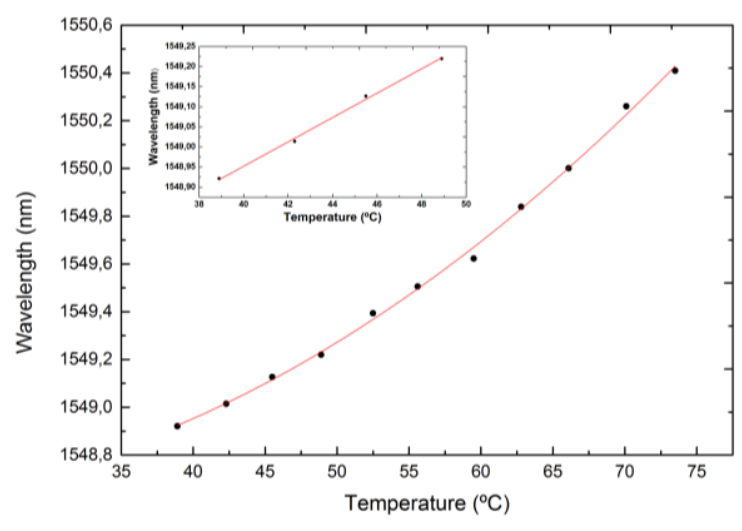

Fig 5. Peak center wavelength response to temperature and linear response in the range $[38.9 ; 48.9]^{\circ} \mathrm{C}$.

In Fig.5, experimental results for temperature measurement are presented. As it can be seen, the registered behavior fits in a polynomial response, which was expected due to the polymer used in the sensor housing. Although, the temperature's range $[38.9 ; 48.9]^{\circ} \mathrm{C}$ experience a linear response with maximum sensitivity of $(30 \pm 1) \mathrm{pm} /{ }^{\circ} \mathrm{C}$. When compared with the FBG unincorporated the sensitivity is very high due to de expansion of the polymer materials.

The last tests are measurements of vibration as a function of wavelength and time, as shown in Fig. 6 and 7 , respectively. It was used a $4 \mathrm{gr}$ weight glued to the sensor's end point to make possible the wanted vibration. The sensor presents a more sensitive response when observed in specific wavelength ranges.

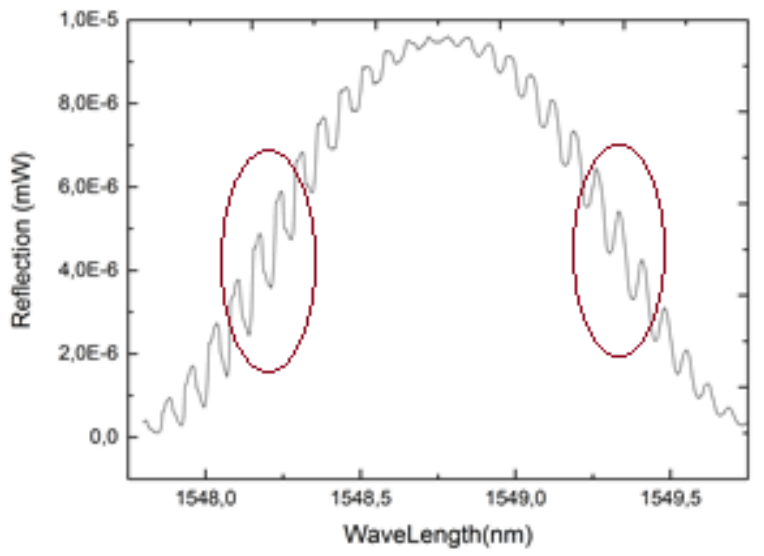

Fig. 6 Effects of vibration on observed spectrum, in reflection, with the most sensitive regions highlighted.

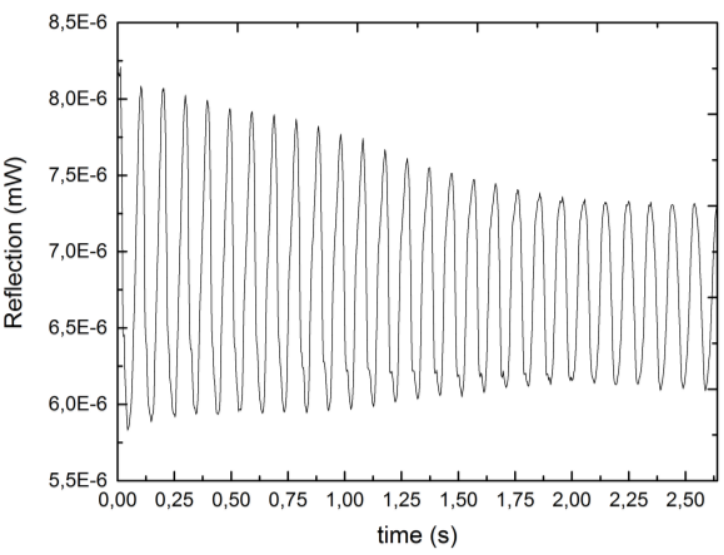

Fig. 7 Effects of vibration on the most sensible range of the spectrum. 
This conclusion is enhanced by the registered zoom out response presented in Fig.6. As shown, the effects of vibration are more noticeable in the range of $[1548.0 ; 1548.5] \mathrm{nm}$ and $[1549.0 ; 1549.5] \mathrm{nm}$. Fig. 7 shows the spectrum for a vibration that was monitored during a period $2.6 \mathrm{sec}$, in the referred wavelengths. From that information it was possible to determine the oscillation frequency value of $10.2 \mathrm{~Hz}$.

\section{CONCLUSIONS}

FBG sensor embedded in 3D printed polymer was proposed. A sensor with cantilever based structure embedded in a polymer 3D printed material was developed. The sensor was submitted to displacement, temperature and vibration. It exhibited maximum displacement sensitivity of $(3.54 \pm 0.15) \mathrm{pm} / \mathrm{mm}$ when applied in the end point, and maximum temperature sensitivity of $(30 \pm 1) \mathrm{pm} /{ }^{\circ} \mathrm{C}$ in the range $[38.9 ; 48.9]{ }^{\circ} \mathrm{C}$. It presented good sensitivity to vibration in certain ranges of the spectral spectrum. For vibration measurement, the fact that the response is more sensitive in certain regions of the spectrum was expected and demonstrated. The spectrum concerning these regions was registered and the oscillation frequency determined was of $10.23 \mathrm{~Hz}$. The cantilever resisted to all the performed tests, only coating's stiffness changed when approaching the polymer's melting point, as expected.

\section{ACKNOWLEDGMENTS}

The involvement in sensorization and the potential capabilities by using new technologies are aligned with previous activities funded by National Foundation for Science and Technology under LAETA Project UID/EMS/50022/2013and presently potentiated with Project NORTE-01-0145-FEDER-000022 - SciTech Science and Technology for Competitive and Sustainable Industries, cofinanced by Programa Operacional Regional do Norte (NORTE2020), through Fundo Europeu de Desenvolvimento Regional (FEDER), which authors gratefully acknowledge. A special thank is also due to Bruno Santos. S. O. Silva received a Pos-Doc fellowship (ref. SFRH/BPD/92418/2013) also funded by the EU and the Portuguese State.

\section{REFERENCES}

[1] K. D. D. Willis, E. Brockmeyer, S. E. Hudson, and I. Poupyrev, "Printed Optics: 3D Printing of Embedded Optical Elements for Interactive Devices," In Proc. of the ACM Symposium on User interface Software and Technology (UIST '12), 589-598 (2012).

[2] A. D. Kersey, M. A. Davis, H. J. Patrick, M. LeBlanc, K. P. Koo, C. G. Askins, M. A. Putman, and E. J. Friebele, "Fiber Grating Sensors," Journal of Lightwave Technology, 15(8),1442-1463 (1997).

[3] Y. L. Lo, "Using in-fiber Bragg-grating sensors for measuring axial strain and temperature simultaneously on surfaces of structures," Opt. Eng., 37(8), 2272-2276 (1998).

[4] G. Cazzulani, S. Cinquemani, and L. Comolli, "Enhancing active vibration control performances in a smart structured by using fiber Bragg grating sensors," Proc. SPIE 8345, Sensors and Smart Structures Technologies for Civil, Mechanical, and Aerospace Systems, 834530 (2012).

[5] T. K. Gangopadhyay, "Prospects for fiber Bragg gratings and Fabry-Perot interferometers in fiber-optic vibration sensing," Sensors and Actuators A, 113(1), 20-38 (2004).

[6] B. A. L. Gwandu, X. W. Shu, Y. Liu, W. Zhang, L. Zhang, and I. Bennion, "Simultaneous measurement of strain and curvature fibre Bragg gratings," Sensors and Actuators A, 96(2-3), 133-139 (2002).

[7] Y. Tan, L. Cai, T. Li, and Q. Wei, "Study on Non-Contact Fiber Bragg Grating Vibration Sensor," In Proc. Of the International Conference on Innovative Design and Manufacturing, 211-216 (2014).

[8] P. Ray, S. Saurabh, U. Srinivasan, and B. Srinivasan, "Fiber Bragg Grating-Based Vibration Sensing for Machine Prognostics," ational Seminar \& Exhibition on Non-Destructive Evaluation, NDE-India 2014 (2014).

[9] C. Schubert, M. C. van Langeveld, and L. A. Donoso, "Innovation in 3D printing: a 3DD overview from Optics to Organs," Br. J. Ophthalmol., 98, 159-161 (2014).

[10] B. Berman, "3-D printing: The new industrial revolution," Business Horizons, 55(2), 155-162 (2012). 